\title{
DIFICULTADES PARENTALES RELACIONADAS CON EL EFECTO DE ESTIGMA EN EL ÁMBITO DE LOS TRASTORNOS GENERALIZADOS DEL DESARROLLO Y ESTRATEGIAS DE INTERVENCIÓN EN FAMILIAS
}

\author{
Ma Carmen Lozano-Segura \\ Ana Manzano-León \\ Cristian Aguilera-Ruíz \\ Carla Casiano Yanicelli \\ Universidad de Almería \\ mcarmenlozseg@gmail.com \\ https://doi.org/10.17060/ijodaep.2017.n1.v3.983
}

Fecha de Recepción: 2 Marzo 2017

Fecha de Admisión: 1 Abril 2017

\section{RESUMEN}

Introducción: Cuando un niño o niña recibe el diagnóstico de TEA su familia sufre un proceso de adaptación a la nueva situación que se afronta. Son muchos los casos de estigmatización en los Trastornos Generalizados del Desarrollo (TGD) y las dificultades que se les plantean a las familias. Por ello se debe dar respuesta a sus dificultades mediante estrategias de intervención útiles.

Objetivos: El objetivo del presente trabajo es estudiar, mediante una revisión sistemática de la literatura científica, las dificultades parentales y el efecto de estigma en el ámbito de los trastornos generalizados del desarrollo, así como las estrategias de intervención en familias de niños con autismo.

Metodología: Se ha realizado una revisión sistemática de artículos científicos consultando las bases de datos. Los materiales han sido restringidos por fecha de publicación (2000/2017) y se han buscado con los siguientes descriptores en español e inglés: estigma y autismo, autismo y familias, terapias en familias y autismo/ Stigma and autism, autism and families, family therapies and autism.

Resultados: Los resultados ponen de manifiesto que hay determinadas conductas parentales consideradas de riesgo para el desarrollo y fomento de las dificultades psicológicas de los hijos, así como las dificultades existentes en el ámbito familiar y escolar relacionado con la estigmatización de los niños diagnosticados de TGD. A lo largo de los años han sido muchas las estrategias de intervención empleadas para paliarlas, siendo las basadas en terapias de tercera generación las que han reportado mejores resultados.

Conclusión: Surge la necesidad de fomentar técnicas propias de la Terapia de Aceptación y Compromiso en escuelas de familias, así como seguir investigando al respecto.

Palabras clave: Dificultades parentales, estigma, TGD y estrategias de intervención. 


\section{INTRODUCCIÓN}

El autismo es un trastorno que se inserta dentro de un grupo de complejos trastornos del desarrollo conocidos como Trastornos Generalizados del Desarrollo (TGD) (Diasnostic and Statistical Manual of Mental Disorders, quinta edición, American Psychiatric Association, 2013). Muchos profesionales y padres, así como en el presente trabajo, se refieren a este grupo como Trastornos del Espectro Autista o TEA. Se estima la presencia del Trastorno del Espectro autista en 2/1,000 individuos. En la actualidad, alrededor de uno de cada 175 niños de todo el mundo nace con este trastorno, aunque la frecuencia es distinta en cada país (Oviedo, Manuel-Apolinar, De la Chesnaye y Guerra Araiza, 2015). El autismo es un trastorno con una gran heterogeneidad de síntomas. Se trata de un trastorno que puede desencadenar diversos tipos de dificultades en la vida cotidiana de la persona, y suponer un reto adicional en el seno de la familia y los centros educativos. Al ser de carácter crónico, estudiado como un continuo de gran espectro, se presentan dilemas sobre cómo actuar desde el ámbito familiar y el ámbito escolar (Gómez, Molina, y Zaldívar, 2005).

Existen diferencias entre el autismo que describe el DSM-V y el DSM-IV. En el segundo no se especifican los aspectos sensoriales, mientras que en el primero si se incluyen. En el DSM-IV se restringe hasta los tres años la edad de aparición máxima del trastorno, y se incluye la mención de aparición de un deterioro clínicamente significativo de diversos aspectos de la vida de quien lo sufre. Finalmente, en la nueva versión se contempla la posibilidad de comorbilidad del autismo con discapacidad intelectual.

\section{OBJETIVOS}

El objetivo del presente trabajo es estudiar, mediante una revisión sistemática de la literatura científica, las dificultades parentales y el efecto de estigma en el ámbito de los trastornos generalizados del desarrollo, así como las estrategias de intervención en familias de niños con autismo.

\section{METODOLOGÍA}

Se ha realizado una revisión sistemática de artículos científicos consultando las bases de datos Web of Science, Scopus e INDAGA. Los materiales han sido restringidos por fecha de publicación (2000/2017) y se han buscado con los siguientes descriptores en español e inglés: estigma y autismo, autismo y familias, terapias en familias y autismo/ Stigma and autism, autism and families, family therapies and autism.

No se han hecho restricciones respecto al tipo de estudio. Se han revisado resúmenes/abstracts y se han leído en profundidad los artículos seleccionados así como todos los artículos con resultados empíricos y teóricos de relevancia para esta revisión.

\section{RESULTADOS}

Es numerosa la evidencia de la literatura respecto al papel que tienen los estilos educativos parentales como factores de riesgo 0 de protección en diferentes problemas psicológicos en la infancia. En la tabla 1 y 2 se hace un repaso de los estudios que han versado sobre los factores de riesgo del entorno familiar en psicopatología de la infancia y la relación entre estilos parentales y trastorno psicológicos específicos respectivamente. 
TABLA 1

Factores de riesgo del entorno familiar en psicopatología en la infancia

\begin{tabular}{|c|c|}
\hline AUTOR & FACTORES DE RIESGO \\
\hline Casera, Sullana y Torrubia (2002). & $\begin{array}{l}\text { Ambiente familiar estresante y conflictivo, } \\
\text { presencia de agresividad y prácticas negligentes o } \\
\text { maltrato infantil }\end{array}$ \\
\hline Bradley (2000). & $\begin{array}{l}\text { Dificultades en la emoción expresada (EE), } \\
\text { marcadas por presencia de críticas y } \\
\text { desaprobación por parte de los padres, falta de } \\
\text { calor afectivo, hostilidad y excesiva implicación } \\
\text { emocional, fomenta en el niño sentimientos de } \\
\text { rechazo y de baja autoestima. }\end{array}$ \\
\hline Ramírez (2007). & $\begin{array}{l}\text { Afecto negativo, control excesivo, castigos no } \\
\text { físicos y énfasis en el logro del niño. }\end{array}$ \\
\hline Gómez (2005). & Intolerancia por parte de los padres al malestar. \\
\hline Bradley (2000) & $\begin{array}{l}\text { Vinculación insegura con un cuidador primario } \\
\text { fomenta la vulnerabilidad a otros factores de } \\
\text { riesgo de alteraciones psicológicas. }\end{array}$ \\
\hline $\begin{array}{l}\text { Ezpeleta (2005); Moreno y Revuelta, } \\
\text { (2002). }\end{array}$ & Conflictos matrimoniales de los padres. \\
\hline
\end{tabular}

Nota. Elaboración propia. 
Tabla 2

Relación entre estilos parentales y trastornos psicológicos específicos

\begin{tabular}{|c|c|}
\hline AUTOR & APORTACIÓN \\
\hline Castro (2005) & $\begin{array}{l}\text { Destaca el papel del funcionamiento familiar en } \\
\text { trastornos disociales, problemas de baja autoestima y } \\
\text { locus de control inadecuado, hostilidad, } \\
\text { impulsividad, dificultades de vinculación afectiva, } \\
\text { dificultades en las relaciones sociales y baja } \\
\text { tolerancia a la frustración, entre otros. }\end{array}$ \\
\hline $\begin{array}{l}\text { Epstein y Sanders (2002), Alonso y } \\
\text { Román (2005), Aunola y Nurmi (2005), } \\
\text { Newcomb, Mineka, Zinbarg y Griffith } \\
\text { (2007). }\end{array}$ & $\begin{array}{l}\text { Estilos educativos parentales (por ejemplo, no } \\
\text { democráticos) influyen en problemas de tipo } \\
\text { internalizante y externalizante en los hijos. También } \\
\text { pueden afectar la baja autoestima en los niños y } \\
\text { dificultades en su rendimiento académico }\end{array}$ \\
\hline Castro (2005). & $\begin{array}{l}\text { Estilos educativos parentales de control y exigencia } \\
\text { sin afecto se asocian a trastornos asociados a la } \\
\text { depresión y la ansiedad, mientras que estilos } \\
\text { educativos excesivamente permisivos, y más si se } \\
\text { acompaña de indiferencia y rechazo, se asocia a } \\
\text { trastornos de conducta en el hijo. }\end{array}$ \\
\hline $\begin{array}{l}\text { Edwards, Barkley, Laneri, Flecher y } \\
\text { Metevia (2001); Moreno y Revuelta } \\
\text { (2002). }\end{array}$ & $\begin{array}{l}\text { Padres irritables y negativos, con ausencia o } \\
\text { disparidad de normas entre los miembros de la } \\
\text { familia se relaciona con niños con trastornos } \\
\text { negativistas/desafiantes o disociales. }\end{array}$ \\
\hline Raya, Pino y Herruzo (2009). & $\begin{array}{l}\text { Estilos educativos diferenciales en el grado de } \\
\text { disciplina, compromiso, satisfacción y nivel de } \\
\text { autonomía en la crianza se ha relacionado con niños } \\
\text { con comportamientos agresivos y trastorno por } \\
\text { déficit de atención e hiperactividad. }\end{array}$ \\
\hline Eisenberg y Valiente (2002). & $\begin{array}{l}\text { Bajos niveles de supervisión, consistencia y } \\
\text { afectividad, así como altos niveles de ira, hostilidad y } \\
\text { coerción se relacionan con problemas externalizantes. }\end{array}$ \\
\hline Eisenberg y Valiente (2002) y Toro, & Los conflictos conyugales \\
\hline
\end{tabular}




\begin{tabular}{|c|c|}
\hline (2005). & $\begin{array}{l}\text { con sintomatología exteriorizada; fundamentalmente, } \\
\text { por la existencia de mayor disciplina coercitiva de los } \\
\text { padres y madres. }\end{array}$ \\
\hline Castro (2005). & $\begin{array}{l}\text { Estilos educativos parentales de control y exigencia } \\
\text { sin afecto se asocia a trastornos asociados a la } \\
\text { depresión y la ansiedad, mientras que estilos } \\
\text { educativos excesivamente permisivos y más si se } \\
\text { acompaña de indiferencia y rechazo se asocia a } \\
\text { trastornos de conducta en el hijo }\end{array}$ \\
\hline $\begin{array}{l}\text { Newcomb, Mineka, Zinbarg y Griffith } \\
\text { (2007). }\end{array}$ & $\begin{array}{l}\text { Destacan el papel de las prácticas de sobreprotección } \\
\text { y la percepción negativa del cuidado y la crianza en } \\
\text { el desarrollo de desórdenes emocionales en la } \\
\text { infancia y la adolescencia como la depresión y la } \\
\text { ansiedad. }\end{array}$ \\
\hline Mulsow (2008). & $\begin{array}{l}\text { Los estilos parentales autoritarios y castigadores } \\
\text { dificultan en el menor el desarrollo de estrategias y } \\
\text { competencias emocionales necesarias para la } \\
\text { adaptación en distintos contextos y situaciones } \\
\text { vitales. }\end{array}$ \\
\hline Bertino, Connell y Lewis (2012). & $\begin{array}{l}\text { Se observan relaciones entre la personalidad de los } \\
\text { padres, concretamente patrones borderline, paranoide } \\
\text { y de evitación, y problemas de conducta } \\
\text { externalizante en los menores. }\end{array}$ \\
\hline
\end{tabular}

Nota. Elaboración propia.

\section{Dificultades parentales y efecto de estigma en el ámbito de los trastornos generalizados del desarrollo:}

Cuando unos padres reciben el diagnóstico de autismo de su hijo son muchos los sentimientos que se agolpan y muchas las formas en las que se gestionan. En primer lugar, el simple hecho de recibir una etiqueta por parte de un profesional tiene un efecto de estigma en el niño que afecta también a sus padres (Gómez et al., 2005). Este efecto estigmatizante no es diferente al de cualquier otro trastorno grave de larga duración, por ello hay que conocerlo bien y estar alerta a cómo puede afectar al pequeño y su familia.

Estigmatizar quiere decir generar efectos contraproducentes en las expectativas y atribuciones de los demás. De hecho, a veces, se tiende a clasificar a la persona, no al comportamiento atípico 0 disfuncional de dicha persona, pudiendo variar la forma de interaccionar con ella: desde atender comportamientos acordes a la etiqueta, en detrimento de otros, hasta generalizar las características 


\section{DIFICULTADES PARENTALES RELACIONADAS CON EL EFECTO DE ESTIGMA EN EL ÁMBITO DE LOS TRASTORNOS GENERALIZADOS DEL DESARROLLO Y ESTRATEGIAS DE INTERVENCIÓN EN FAMILIAS}

del comportamiento disfuncional o problemático a otros repertorios. Así se produce la llamada "profecía autocumplida" (Río, 2015), es decir, la etiqueta se vuelve circular y se produce iatrogenia.

Otra evidencia empírica, posible ante el etiquetaje, es que cuando el pequeño diagnosticado acude a un centro educativo los profesores pueden hacer atribuciones causales diferentes ante situaciones de éxito y de fracaso. Suelen tener bajas expectativas respecto a los niños etiquetados; sucede pues que ante las etiquetas los profesores atribuyen los déficits y excesos del niño a algo crónico, estable e inmutable (locus interno y estable). Este hecho resulta ser una dificultad añadida para el aprendizaje del menor, produciendo el efecto del etiquetaje factores similares a los mostrados por historias continuadas de fracaso escolar (De la Torre y Godoy, 2002).

Se conoce que el efecto del etiquetaje en niños con autismo repercute también en sus familiares más directos, en especial en los padres, que tratan a sus hijos de acuerdo a la etiqueta produciéndose la anteriormente citada "profecía autocumplida", pero aún son escasos los estudios que versan sobre esta temática tan concreta. Los que sí se han estudiado ampliamente han sido los efectos inmediatos y a medio plazo de la comunicación del diagnóstico de autismo a los padres, cómo se gestionan las emociones y cómo se actúa.

Verdaderamente nadie está preparado para recibir un diagnóstico de autismo de un hijo. En general las primeras emociones que surgen están asociadas a las etapas de duelo, y es posible que en un futuro reaparezcan de vez en cuando (Tijeras, Fernández-Andrés, Pastor-Cerezuela, SanzCervera, Vélez, Blázquez-Garcés y Tárraga, 2015). Siguiendo esta última fuente, una reacción inicial típica es ignorar la noticia o simplemente rechazar el diagnóstico. Para superarlo es fundamental confrontar y aceptar aquello que se está sintiendo y permitirse a uno mismo sentir tristeza (Luciano y Gutiérrez, 2001). Con el tiempo la tristeza deja paso a la rabia, que puede manifestarse de muchas maneras: con irritabilidad hacia otros, reaccionando excesivamente, gritando. La rabia es una reacción saludable y esperada ante la sensación de pérdida y el estrés que acompaña a este diagnóstico, y soltar esa rabia ayuda a liberar tensión (Tijeras et al., 2015).

Alternándose con la rabia los padres atraviesan periodos de negación de los que generalmente no son conscientes (Artigás et al., 2010). Durante estos periodos les cuesta escuchar información relacionada con el diagnóstico de su hijo. Es importante aquí que aprendan a aceptar que se está pasando por un periodo de negación para no perder nunca de vista la importancia del tratamiento de su hijo. Puede venir acompañado también de agresividad, sobre todo frente a los profesionales que tratan de ayudarlos (Tijeras et al., 2015).

Esta primera fase da paso a un sentimiento de soledad. Las familias suelen apreciar que la nueva situación a la que se enfrentan no les va a dejar tiempo para llevar a cabo una vida social como antes y que sus amigos y familiares no van a entender la situación.

Por último, se llega a la aceptación del diagnóstico (Artigás el al., 2010). Esto simplemente significa que los padres ya están preparados para luchar por su hijo. De vez en cuando vuelven a experimentar angustia, otras veces será impotencia y rabia de que el autismo haya causado que sus vidas sean muy diferentes a lo que habían planeado. Pero también aparecerá la esperanza al ver mejorar a su hijo con las intervenciones oportunas.

Para llegar a ese estado de aceptación se aconseja a los padres entrar en acción cuanto antes, es decir, que su hijo comience un tratamiento. Se aconseja también que tengan cerca a personas de confianzas con quien hablar (Artigás et al., 2010).

\section{Estrategias tradicionales de intervención con familias desde la Terapia de Conducta}

A la hora de intervenir con los padres, la estrategia de dar instrucciones a los padres sobre lo que deben hacer o cambiar la interacción con sus hijos fue la que acaparó las terapias en un primer 
momento. Se instruía a las familias para que actuasen de la forma señalada por el terapeuta, bien mediante instrucciones verbales en una relación bis a bis, en charlas o conferencias, o por escrito mediante pautas o sugiriendo lecturas de autoayuda. Esta metodología se solía complementar con dinámicas de grupo heterogéneas a las que asisten tanto padres de niños con o sin desórdenes psicológicos (Gómez, García-Barranco, Hodar y Martínez, 2012). Por tanto, la intervención mediante la instrucción es más bien prevención primaria y secundaria, que no invita a la reflexión, sino que es un modelo directivo.

El siguiente tipo de terapia se centra en las contingencias, especialmente en la discriminación del propio comportamiento 0 autoconocimiento, teniendo como base la investigación del papel de la conducta verbal. Se trata de establecer una relación bis a bis entre padres y profesionales, en la que se va planteando cuestiones que pongan en duda algunas de sus actuaciones 0 creencias o que sirvan como peldaños para llegar a la solución de los conflictos con sus hijos (método socrático); con esas ayudas o señales de tipo verbal -pistas-, cuando los padres lleguen a establecer o describir la actuación futura que consideramos que "deben hacer" se ha de valorar efusivamente sus conclusiones mediante reforzamiento positivo (Vargas y Ramírez, 2012). En este tipo de estrategias, al igual que en el caso anterior, consideran que el problema es que el profesional valora según su criterio lo que está bien o mal, sin tener un componente claro de discernir valores y reflexionar sobre ellos.

Otra forma clásica de intervención con familiar es proporcionar modelos de actuaciones y pensamientos correctos. Pueden ser dados a los padres en diferentes soportes: mediante la presentación de vídeos representativos de la problemática tratada y de la actuación de los padres; mediante el análisis de ensayos clínicos o estudios de casos; mediante las descripciones de las formas de actuar de otros padres que intervienen en una dinámica de grupo; o mediante modelos en vivo en los que el profesional u otra persona actúa (con o sin descripción paralela de lo que hacen) y los padres lo ven, tratándose de la táctica más común en el entrenamiento de autonomía personal, atención temprana o control de comportamientos perturbadores graves (Gómez et al., 2012). Se trataría pues de un modelado típico de modificación de conducta; por tanto, en esta variedad tampoco entran en juego valores personales.

Un último ejemplo clásico de intervención ampliamente utilizada es la aplicación directa de contingencias, es decir, reforzar las actuaciones correctas de los padres y corregir o rectificar las incorrectas. Es especialmente útil en control de agresivas, entrenamiento de habilidades sofisticadas y de vital importancia en la estimulación temprana en retraso mental y autismo. Estas contingencias pueden ser dadas bien de manera directa 0 in situ o bien descritas a raíz de la descripción de las actuaciones objeto de valoración. Siempre aparecen combinadas con alguna de las tácticas anteriores. Esta táctica en la literatura se incluye dentro de lo que se denomina "demostrar el dominio", de diferentes formas: en vivo, por ejemplo en la casa a través de visitas al hogar; a través de roleplaying, en el que en un contexto simulado se le plantea a los padres una serie de problemas sobre los que han de cambiar sus actitudes y reacciones; o mediante grabaciones de los padres de sus interacciones con los hijos en casa que después llevan a los terapeutas para corregir y reforzar (Vargas y Ramírez, 2012).

\section{Evolución en la terapia}

La evolución de la terapia ha sido progresiva y cambiante a los largo de los años. En la actualidad se considera efectiva la terapia a través de una Escuela/Taller para padres de niños con autismo que se basa en los fundamentos de la Terapia de Aceptación y Compromiso (Blackledge, Hayes, y Steve, 2006) que a su vez se enmarca en las Terapias de Tercera Generación (Pérez, 2006). 


\section{DIFICULTADES PARENTALES RELACIONADAS CON EL EFECTO DE ESTIGMA EN EL ÁMBITO DE LOS TRASTORNOS GENERALIZADOS DEL DESARROLLO Y ESTRATEGIAS DE INTERVENCIÓN EN FAMILIAS}

Esta forma de trabajar con padres de niños con dificultades es novedosa, habiendo sido otras intervenciones clásicas, mencionadas anteriormente, las más frecuentes.

Las terapias de Tercera Generación suponen la recuperación de las raíces contextuales de la terapia de conducta (Pérez, 2006). Con esta tercera generación de terapias se da un replanteamiento contextual de la psicología clínica, donde se cuestiona la visión clásica de ver los trastornos mentales según el foco del modelo médico. La aportación de la Tercera Generación concierne a la psicopatología, ofreciendo alternativas a las categorías de los trastornos al uso como, por ejemplo, el Trastorno de Evitación Experiencial (Luciano, Valverde y Rodríguez, 2005), ofreciendo el análisis funcional como apto también para los eventos privados y para el tratamiento. La Tercera Generación defiende el abandono de la lucha contra los síntomas y en su lugar la reorientación de la vida en términos de orientación a los valores importantes para cada persona, y es en este punto donde la Terapia de Aceptación y Compromiso (ACT) se convierte en abanderada de estos principios y se conforma como una manera de hacer terapia novedosa que paradójicamente no plantea una filosofía nueva de vida (ya la plantearon grandes pensadores a lo largo de la historia), siendo la más completa de las denominadas terapias de tercera generación o contextuales (Luciano, Valdivia, Gutiérrez y Páez-Blarrina, 2006).

ACT parte de un marco global de referencia sobre las ventajas y desventajas de la condición humana, manteniendo una filosofía contextual-funcional, y por tanto, asume los presupuestos sobre el impacto de las contingencias. Es coherente con un modelo funcional sobre la cognición y el lenguaje (la Teoría del Marco Relacional) y sustenta una perspectiva nueva de la psicopatología en la que es central el concepto funcional de evitación experiencial destructiva. Resalta así la conexión entre investigación básica, psicopatología y la alteración de los trastornos psicológicos.

Para ACT, la base de los problemas psicológicos está en la transformación de funciones: sin experiencia directa un determinado estímulo puede resultar aversivo (o apetitivo) por su participación en un marco relacional. Los seres humanos somos seres verbales regidos por reglas verbales que hemos ido incorporando y creando a lo largo de nuestra vida, y a la hora de desenvolvernos en ella nos regimos a través de esas reglas. Sin embargo, las reglas verbales pueden promover una insensibilidad a las consecuencias directas de una conducta, creando también patrones de inflexibilidad, y en esas situaciones deberíamos debilitar el control verbal tan fuerte que guía nuestra conducta. La mejor manera de debilitar el control verbal sería alterar el contexto en que ocurre, no su contenido. Así, ACT defiende que los pensamientos no deben ser jamás alterados, al contrario, propone su aceptación. Defiende que en la condición humana hay cabida para todo tipo de sentimientos y emociones, que debemos tomar contacto con ello, creando una distancia (el pensamiento y yo como algo diferente) y no rechazarlos, y cambiar nuestra regla verbal inculcada socialmente: "si me siento mal no puedo funcionar", por una nueva regla: "Me siento mal, tomo contacto con ello, lo acepto, y sigo con mi vida, con aquello que es importante para mí". Busca flexibilizar la reacción de malestar disminuyendo el control del repertorio verbal para que la persona pueda seguir el camino de acuerdo con sus valores. Por lo tanto, para llevar a cabo una terapia basada en ACT es fundamental que se sigan unos pasos claros donde la clarificación de valores tiene un papel principal, y ello se trabaja con la ayuda de metáforas y ejercicios simbólicos (Vargas y Ramírez, 2012).

\section{CONCLUSIONES}

La combinación de algunas de las estrategias de intervención nombradas en este trabajo para trabajar con familias ha sido usada con una elevada efectividad y se conocen a nivel aplicado como muestras ilustrativas en diferentes centros 0 instituciones. No obstante, como programas globales de entrenamiento no han sido estudiados a nivel experimental, ni han sido validados los diferentes 
componentes de estos paquetes de intervención de manera aislada y comparativa de una manera contundente, Sólo a través de ciertos estudios clínicos o correlacionales pero con limitaciones y variabilidad, o que son simplemente protocolos de uso limitado en las instituciones o centros (Gómez et al., 2012). Por tanto, se extrae como conclusión que es necesario aumentar los estudios de metaanálisis en las intervenciones en y con familias.

Aunque estas terapias existían desde un principio en la intervención en familias de niños con autismo y, en mayor o menor medida, están validadas, la revolución en este ámbito surgió con el auge de las terapias de tercera generación, y fundamentalmente con el avance de la Terapia de Aceptación y Compromiso (Luciano et al., 2006).

Es ampliamente conocido que los estilos educativos de los padres afectan al desarrollo de sus hijos (Lozano, Galián y Huéscar, 2007), y una vez revisadas las barreras más frecuentes que les surgen a los padres tras el diagnóstico, es importante saber si los estilos educativos de padres de niños con autismo siguen la línea de aceptación y compromiso, si se diferencian en algo de los estilos de padres de niños sin autismo, así como conocer dificultades concretas, o puntos que funcionan como barreras psicológicas para poder ayudarles a seguir el camino que lleve a la implicación hacia sus hijos, teniendo la mayor calidad de vida posible.

\section{REFERENCIAS}

Alonso, J., y Román, J.M. (2005). Prácticas educativas familiares y autoestima. Psicothema, 17(1), 76-82

American Psychiatric Association-APA- (2013). Diagnostic and statistical manual of mental disorders (5th ed.). Arlington, VA: American Psychiatric Publishing.

American Psychiatric Association -APA- (2000). Diagnostic and statistical manual of mental disorders (DSM-IV-TR). Arlington, VA: American Psychiatric Publishing.

Aunola, K. y Nurmi, J.E. (2005). The Role of Parenting Styles in Children's Problem Behavior. Child Development, 76, 1144-1159.

Blackledge, J. T., y Hayes, S. C. (2006). Using acceptance and commitment training in the support of parents of children diagnosed with autism. Child and Family Behavior Therapy, 28(1). https://doi.org/10.1300/J019v28n01 01

Bradley, M. (2000). Emotion and motivation. En J. T. Caccioppo, L. G. Tassinary y G. G. Bernstson (Eds.), Handbook of Psychophysiology (pp. 602-642). Cambridge: University Press.

Casera, X., Fullana, M., y Torrubia, R. (2002). El trastorno social. En M. Servera. Intervención en los trastornos del comportamiento infantil. Una perspectiva conductual de sistemas. Madrid: Pirámide.

Castro, A. (2005). Alfabetización emocional: la deuda de enseñar a vivir con los demás. Revista Iberoameticana de Educación, 15. ISSN: 1681-5653.

Cerezuela, G. P. (2014). Estrés y afrontamiento en familias de hijos con trastorno de espectro autista. International Journal of Developmental and Educational Psychology. INFAD Revista de Psicología, 4, 425-434.

De la Torre, C., y Godoy, A. (2002). Influencia de las atribuciones causales del profesor sobre el rendimiento de los alumnos. Psicothema, 14(2), 444-449.

Recuperado de: http://www.redalyc.org/articulo.0a?id=282130698004. (2014).

Epstein, J. y Sanders, M. (2002). Family, school, and community partnerships. M Bornstein M (Ed). Handbook of parenting: Vol. 5. Practical issues in parenting. Erlbaum; Mahwah, NJ. pp. 507-437

Ezpeleta, L. (2005). Factores de riesgo en psicopatología del desarrollo. Barcelona: Masson. 


\section{DIFICULTADES PARENTALES RELACIONADAS CON EL EFECTO DE ESTIGMA EN EL ÁMBITO DE LOS TRASTORNOS GENERALIZADOS DEL DESARROLLO Y ESTRATEGIAS DE INTERVENCIÓN EN FAMILIAS}

ISBN: 84-458-1489-3

Gómez et al. (2005). El "bullying" y otras formas de violencia adolescente. Cuad Med Forense, 13.48-49.

Gomez, I., García-Barranco, R., Hodar, J.C.P., y Martínez, M.M. (2012). Intervención en valores con familias de riesgo social desde la Terapia de Aceptación y Compromiso. Análisis y Modificación de Conducta, 38, 157-158.

Gómez, I., Molina, A. y Zaldivar, F. (2005). Una Revisión sobre los Posibles Efectos latrogénicos de la Clasificación Diagnóstica en el Ámbito Infantil y Alternativas de Corte Funcional. Revista Mexicana de Psicología, 22,69-78.

Lozano, E. A., Conesa, M. D. G. y Hernández, E. H. (2007). Relaciones entre estilos educativos, temperamento y ajuste social en la infancia: Una revisión. Anales de Psicología, 23(1), 33-40.

Luciano, M. C. y Valdivia, S. (2006). La Terapia de Aceptación y Compromiso (ACT): fundamentos, características y evidencia. Papeles Del Psicólogo, 27(2), 79-91. Recuperado de http://0-dialnet.unirioja.es.cataleg.uoc.edu/servlet/articulo?codigo $=2009007 \&$ orden $=86760 \&$ info $=\operatorname{link} \% 5 \mathrm{C}$ nhttp://0-dialnet.unirioja.es.cataleg.uoc.edu/servlet/extart?codigo=2009007

Luciano, M.C., Valdivia, S.,Gutíerrez, 0. y Páez-Blarrina, M. (2006). Avances desde la Terapia de Aceptación y Compromiso (ACT). eduPsykhé. 5, 173-201.

Luciano, M. C., y Gutiérrez, 0. (2001). Ansiedad y Terapia de Aceptación y Compromiso. Análisis y Modificación de Conducta. 27, 373-398.

Luciano, M.C., Gutiérrez, 0. y Rodríguez, M. (2005). Análisis de los contextos verbales en el trastorno de evitación experiencial y en la terapia de aceptación y compromiso. Revista latinoamericana de psicología, 37, 333-358.

Moreno I y Revuelta F (2002). El trastorno por negativismo desafiante. En M Servera (Ed.) Intervención en los trastornos del comportamiento infantil. Una perspectiva conductual de sistemas. (pp. 255-276). Madrid: Pirámide.

Newcomb, K.; Mineka, S.; Zinbarg, R. y Griffith, J. (2007). Perceived Family Environment and Symptoms of Emotional Disorders: The Role of Perceived Control, Attributional Style, and Attachment. Cognition Therapy Research 31:419-436.

Oviedo, N., Manuel-Apolinar, L., de la Chesnaye, E., y Guerra-Araiza, C. (2015). Aspectos genéticos y neuroendocrinos en el trastorno del espectro autista. Boletín Médico Del Hospital Infantil de México, 72(1), 5-14. https://doi.org/10.1016/j.bmhimx.2015.01.010

Pérez Álvarez, M. (2006). La terapia de conducta de tercera generación. EduPsykhé: Revista de Psicología Y Psicopedagogía, 5(2), 159-172. https://doi.org/10.5093/cc2010v1n1a6

Río, M.A., (2015). Procesos de etiquetaje y escuela. RASE (Revista de La Asociación de Sociología de La Educación), 8(3), 310-449.

Ramírez, M. S. (2007). Del trabajo en redes a la reflexión e investigación de objetos de aprendizaje. Tema uno del Simposio Objetos de aprendizaje como recursos digitales de enseñanza: redes, desarrollos e investigación. Conferencia Internacional en Tecnología e Innovación Educativa, REDIIEN'07. Monterrey, México.

Sánchez-Raya, M. A., Martínez-Gual, E., Elvira, J. A. M., Salas, B. L., y Cívico, F. A. (2015). La atención temprana en los trastornos del espectro autista (TEA). Psicología Educativa, 21(1), 55-63. https://doi.org/10.1016/j.pse.2014.04.001

Tijeras, A., Fernández-Andrés, M.I., Pastor-Cerezuela, G., Sanz-Cervera, P., Vélez, X., BlázquezGarcés y Tárrega, R. (2016). Estrategias y estilos de afrontamiento parental en familias con niños con Trastorno de Espectro Autista (TEA) con o sin Trastorno por Déficit de Atención con Hiperactividad (TDAH). International Journal of Developmental and Educational Psychology. Revista INFAD de Psicología., 2(1), 311-322. 
LA PSICOLOGÍA HOY: RETOS, LOGROS Y PERSPECTIVAS DE FUTURO. PSICOLOGÍA INFANTIL

Vargar, L.F. y Ramírez, F., (2012). Terapia de Aceptación y Compromiso: Descripción general de una aproximación con énfasis en los valores personales. Rev. Ciencias Sociales Universidad de Costa Rica, (138), 101-110 
